\title{
THE USE OF THE SAME SIZE HOST AND DONOR TREPHINE IN PENETRATING KERATOPLASTY FOR KERATOCONUS
}

\author{
R. R. GOBLE, S. J. HARDMAN LEA and M. G. FALCON \\ London
}

\begin{abstract}
SUMMARY
The results of a retrospective analysis of 49 eyes $(40$ patients) that underwent penetrating keratoplasty for keratoconus are presented. All grafts had the same size trephine for both host and donor corneas. Ninety-eight per cent of eyes achieved a post-operative visual acuity of $6 / 12$ or better; $43 \%$ of eyes attained this level of acuity unaided, although more than half of these required spectacles or contact lenses to reach their best corrected acuity. The average post-operative spherical ametropia was $\mathbf{- 0 . 5}$ dioptre (SD 2.97 dioptres) and the average postoperative cylinder was -3.8 dioptres (SD 2.63 dioptres). Three eyes $(6 \%)$ required keratorefractive surgery to reduce astigmatism. Wound integrity was satisfactory using the same (size) trephine for both host and donor, with no patient suffering a post-operative wound leak or iris prolapse. The reduction of post-operative myopia and astigmatism is discussed.
\end{abstract}

Keratoconus is one of the commonest indications for penetrating keratoplasty. ${ }^{1.2}$ The use of an oversize donor button in phakic eyes was advocated to reduce the incidence of wound leakage, because donor corneas cut from the endothelial surface are smaller than the opening produced by the same size trephine on the epithelial surface of the host cornea. ${ }^{3.4}$ Although success with oversize donor buttons in terms of graft clarity is high, post-operative myopia and astigmatism may mar an otherwise excellent result. Recent articles have shown that the use of the same size donor and host trephines produces less post-operative myopia than is found in keratoconus patients who receive oversize donor trephines. ${ }^{5-8}$ We investigated the results of 49 eyes grafted for keratoconus which received the same size donor button as host trephine. These grafts were carried out at St Thomas" Hospital between September 1981 and September 1991.

From: South Wing Eye Department. St Thomas" Hospital. London SE1 7EH. UK.

Correspondence to: Mr R. R. Goble. Sussex Eye Hospital. Eastern Road, Brighton BN2 5BF. UK.

\section{MATERIALS AND METHODS}

Details of patients who had undergone penetrating keratoplasty were obtained from operating theatre records and those with keratoconus had their notes retrieved and the following information extracted: age at operation, indication for surgery, number of previous grafts, preoperative visual acuity, surgeon, size of trephine(s) used, suture material and technique, complications during and after surgery, best post-operative acuity and the method of optical correction, time taken to reach $6 / 12$ or better with the method of correction, refraction and length of follow-up.

Donor material prior to 1987 was stored in either a moist chamber or McCarey-Kaufman medium. Since 1987 all grafts have been those provided by the organ storage system (UKTS Bristol).

Surgical techniques varied a little between different surgeons, but all grafts were initially marked with a trephine (without a handle/freestanding), the cut was deepened with a blade and completed with scissors. With the exception of five grafts (four of $7.0 \mathrm{~mm}$ and one of $8.5 \mathrm{~mm}$ ) the diameter of the trephines used was either 7.5 , 7.75 or $8.0 \mathrm{~mm}$. After temporary $7 / 0$ silk overlay sutures and four temporary interrupted 10/0 nylon sutures, a continuous $10 / 0$ nylon suture was used to secure all of the grafts. Topical steroids were normally used for 12 months post-operatively. Removal of the continuous suture earlier than 12 months after surgery was only undertaken in an attempt to reduce high astigmatism or as a result of premature suture loosening.

\section{RESULTS}

A total of 238 eyes underwent penetrating keratoplasty between 1981 and 1991, 37 of which had combined cataract surgery. Fifty-three grafts (22\%) were performed in patients with keratoconus. Forty-nine grafts in 40 patients had the same size trephine used for host and donor corneas. The average age of these 40 keratoconus patients at

Eye (1994) 8, 311-314 C 1994 Royal College of Opithalmokgints 
operation was 25.7 years (range 17-39 years). The main indications for surgery were contact-lens-related in 32 $(65 \%)$, due to corneal scarring in $15(31 \%)$ and resulted from a previously failed graft in $2(4 \%)$. Three consultants performed $78 \%$ of the surgery, M.G.F. being responsible for 35 cases; the remaining $22 \%$ were operated on by supervised surgeons in training. Follow-up ranged from 12 to 116 months with an average of 48 months. There were $4(8 \%)$ graft rejections, 3 of which were successfully treated. Seven patients required a second surgical procedure: 3 were keratorefractive operations to reduce astigmatism, 1 was to remove a steroid-induced cataract and 3 grafts needed resuturing following premature suture loosening, although no patient suffered a wound leak or iris prolapse.

\section{Visual Acuity}

Pre-operative and best corrected post-operative visual acuity are shown in Fig. 1. Best corrected post-operative visual acuity was measured after the dispensing of any optical aids and the method of refractive correction used to reach the best post-operative visual acuities is shown in Fig. 2. Ninety-eight per cent (48 of 49 eyes) achieved 6/12 or better. The one eye not achieving this level of acuity was the unsuccessfully treated graft rejection. Only 1 patient's best corrected visual acuity deteriorated by more than 1 line of Snellen acuity during the study period, and this was due to a steroid-induced cataract which followed the successful treatment of a graft rejection episode.

Another measure of visual outcome was the time taken to achieve $6 / 12$ or better vision either with a prescribed optical aid or unaided. The average time to achieve this level of vision was 5.5 months (SD 4.1 months). The method of optical correction used is shown in Fig. 3. Spectacles or contact lens were prescribed earlier than 6 months post-operatively only if the patient was dissatisfied with his or her uncorrected acuity.

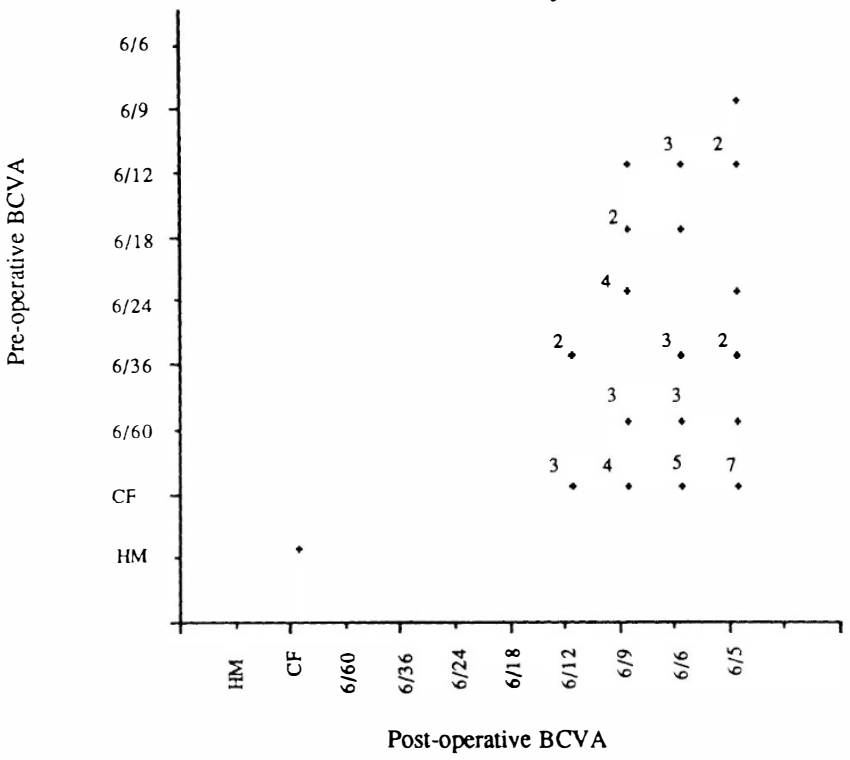

Fig. 1. Comparison of pre-operative and post-operative best corrected visual acuity (BCVA). Numbers are shown where several points coincide.

\section{Refractive Results}

Eight of the 49 cases were never refracted as 7 patients were satisfied with their unaided vision (two $6 / 12$, one $6 / 6$ and four $6 / 5$ ) and the other case was the unsuccessfully treated graft rejection. The refraction which followed suture removal has been used in those patients who underwent this procedure between 6 and 12 months post-operatively in order to try to reduce high astigmatism. All refractions have been transposed to a negative cylinder. The average cylinder was 3.8 dioptres (SD 2.63 dioptres, range -0.50 to -14.00 ). The average spherical ametropia was -0.5 dioptre (SD 2.97 dioptres). The range of spherical ametropia (not spherical equivalent) was from -7.50 to +5.00 dioptres with $31 \%, 43 \%$ and $26 \%$ of patients having,+- and no spherical errors respectively.

Large changes in refraction may occur on removal of the continuous suture even a number of years post-operatively and patients may require new optical aids to be dispensed." The patient with the -14.00 cylinder was one of the 3 who could not be satisfactorily fitted with contact lenses after suture removal and who required tightening sutures and circumferential relieving incisions in order to obtain an acceptable visual correction. This compares with $18 \%$ of the Moorfields Eye Hospital study and $8 \%$ of the East Grinstead study who underwent keratorefractive surgery post-operatively. ${ }^{(1) .11}$

\section{DISCUSSION}

The use of an oversize donor button in a phakic eye undergoing penetrating keratoplasty is to produce the best fit and to minimise the possibility of post-operative wound leakage, because donor corneas cut from the endothelial side are estimated to be an average of $0.26 \mathrm{~mm}$ smaller than the opening produced by the same trephine in the host cornea. ${ }^{3 .+}$ In this series there were no post-operative wound leaks despite the use of the same size host and donor trephines and no patient suffered an iris prolapse, although 3 patients $(6 \%)$ developed premature suture loosening at 2 days, 4 weeks and 6 weeks respectively ( 1 case followed blunt trauma). The practice of graft resuturing for early loosening is mentioned, although its inci-

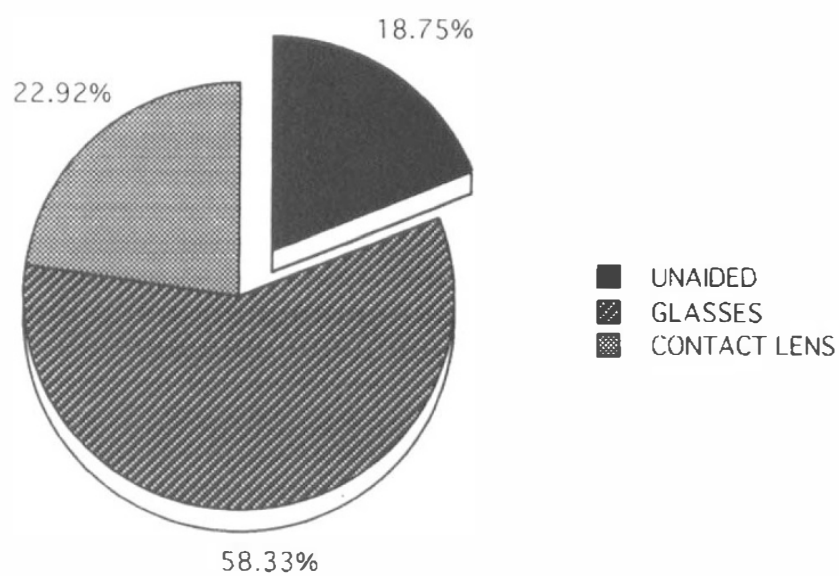

Fig. 2. Method of optical correction to achieve best corrected visual acuity: unaided $(18.75 \%)$. glasses $(58.33 \%)$ or contact lenses $(22.92 \%)$. 


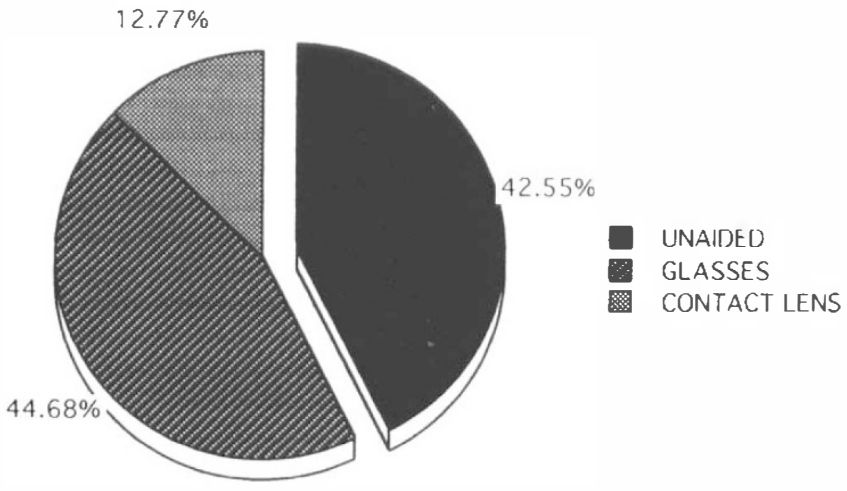

Fig. 3. Method of optical correction to achieve $6 / 12$ or better visual acuity: unaided (42.55\%), glasses $(44.68 \%)$ or contact lenses (12.77\%).

dence is not quoted by other authors who use oversize grafts. ${ }^{10}$ It is therefore difficult to use this particular complication as a comparative measure of wound integrity. Previous studies of keratoconus grafts using the same size host and donor trephines and even small numbers with undersize grafts have reported no problems with wound integrity. $7,12.13$

The refractive results of this study compare favourably with those of other studies in which the surgery has been performed by multiple surgeons including those in training. Eighty-one per cent of Sharif \& Casey's ${ }^{10}$ patients and $91 \%$ of Kirkness et al. ${ }^{11}$ achieved $6 / 12$ or better vision post-operatively, as did $98 \%$ of ours, although these previous studies were larger and spanned two decades. We chose to study the last 10 years, as the protocol of this unit during this time has been to use the same size host and donor trephines and to secure the grafts with a single continuous 10/0 nylon suture. Both of the above studies used oversize donor buttons and $19 \%$ and $11 \%$ respectively reached $6 / 12$ or better unaided. A higher proportion $(43 \%)$ of our patients achieved this level of vision. This may relate to our use of the same size host and donor trephines as there is evidence that this practice reduces postoperative myopia. ${ }^{5-8}$

Myopia in keratoconus may be due to both the excessive corneal curvature and an abnormally increased axial length. Lanier et al. ${ }^{5}$ found that the myopia in 157 unoperated and 66 grafted keratoconus patients was principally corneal as the mean axial length did not differ significantly from that of emmetropic controls. ${ }^{5}$ However, Tuft et al. ${ }^{6}$ noted that post-operative myopia in 60 keratoconus patients who received oversized grafts was mainly the result of abnormally elongated posterior segments, rather than oversteep corneal curvatures. Tuft et al. calculated that even if excessive corneal power was reduced by eliminating the disparity between graft and host, a mean spherical equivalent of -2.8 dioptres would remain. Our results showed a mean post-operative spherical ametropia of -0.5 dioptre with an average cylindrical correction of -3.8 dioptres.

It is usually desirable to minimise post-operative myopia, particularly as grafting in keratoconus is almost always done at an age when the patient has plenty of accommodative reserve. This can be achieved by the use of the same size donor and host trephines which reduces corneal curvature post-operatively. ${ }^{7.8}$ Undersized grafts have an even more marked effect, but may run the risk of excessive post-operative hypermetropia.? Surgery may also have the effect of shortening the axial length of the eye by reducing the depth of the anterior chamber. Lanier et al. ${ }^{5}$ showed an average reduction in post-operative axial length of $0.46 \mathrm{~mm}$ in 8 patients receiving the same size host and donor trephines. This was not significant in such a small sample, although an average reduction of $0.91 \mathrm{~mm}$ in the 8 patients with $0.3 \mathrm{~mm}$ undersized donor buttons was. They estimated that by using undersized buttons, axial myopia may be reduced by $1.25-2.25$ dioptres. Girard et al. ${ }^{13}$ noted that the use of undersized grafts produced the greatest reduction in post-operative myopia, principally by corneal flattening rather than reduction of axial length.

An oval wound in the host cornea is produced by tilting of the trephine, uneven downward pressure or unwanted side-to-side movements of the trephine during cutting, and is one of the main causes of post-operative astigmatism. ${ }^{1+}$ It is desirable to reduce any distorting pressures on the ectatic corneal tissues during cutting of the host tissues, and stay sutures should be put at minimal tension with the trephine (freestanding) simply being used to mark the cornea rather than to cut any significant depth of stroma. Comparative studies of different trephination methods show that the use of a freestanding trephine rather than a trephine with a handle gives significantly better results with less asphericity and a more vertical cut. ${ }^{15.16}$ Numerous complementary surgical techniques have been advocated to minimise astigmatism, such as the positioning of the trephine in relation to the cone and avoidance of any tilting of the trephine. ${ }^{1+.17 .18}$ Cautery to the cone was used by Girard et al. ${ }^{13}$ in order to flatten the cornea and presumably thus reduce the irregular astigmatism prior to trephination. The tension in the continuous suture needs to be greater when the same size host and donor trephines are used in comparison with the tension in an equivalent suture used to secure an oversized button. The suture tends to compress the areas of floppy, ectatic host cornea more than the peripheral areas which are more normal, and this may also have the desirable effect of reducing the irregular astigmatism.

In this retrospective analysis the use of modern microsurgical techniques has given satisfactory wound integrity and the worry of wound disparity is apparently unfounded. This study suggests that the use of the same (size) trephine for cutting both the donor (from the endothelial surface) and the host gives better visual results in penetrating keratoplasty for keratoconus. This technique appeared to reduce the degree of residual myopia. In an attempt to reduce post-operative astigmatism we would recommend that all distorting pressures on the cornea be minimised during cutting of the host cornea and would advocate the use of a freestanding trephine blade for this purpose. 
We would like to acknowledge the help of Mrs S. Singh in retrieving notes and thank $\mathrm{Mr}$ M. G. Kerr-Muir for allowing us to use his patients in this study.

Key words: Graft, Keratoconus. Penetrating keratoplasty.

\section{REFERENCES}

1. Morris RJ, Bates AK. Changing indications for keratoplasty. Eye 1989;3:455-9.

2. Sharif KW, Casey TA. Changing indications for penetrating keratoplasty, 1971-1990. Eye 1993;7:485-8.

3. Foulks GN, Perry HD. Dohlman CH. Oversize corneal donor grafts in penetrating keratoplasty. Ophthalmology 1979;86:490-4.

4. Olson RJ. Variation in corneal graft size related to trephine technique. Arch Ophthalmol 1985;103:1807-11.

5. Lanier JD, Bullington RH, Prager TC. Axial length in keratoconus. Cornea 1992;11:250-4.

6. Tuft SJ, Fitzke FW, Buckley RJ. Myopia following penetrating keratoplasty for ketatoconus. $\mathrm{Br} \mathrm{J}$ Ophthalmol 1992;76:642-5.

7. Wilson SE, Bourne WM. Effect of recipient-donor trephine size disparity on refractive error in keratoconus. Ophthalmology 1989;96:299-305.

8. Javadi MA, Mohammadi MJ, Mirdehghan SA, Sajjadi SH. A comparison between donor-recipient corneal size and its effect on the ultimate refractive error induced in keratoconus. Cornea 1993;12:401-5.

9. Mader TH, Yuan R, Lynn MJ, Stulting RD, Wilson LA, Waring GO. Changes in keratometric astigmatism after suture removal more than one year after penetrating keratoplasty. Ophthalmology 1993;100:119-26.
10. Sharif KW, Casey TA. Penetrating keratoplasty for keratoconus: complications and long-term success. Br J Ophthalmol 1991;75:142-6.

11. Kirkness CM, Ficker LA, Steele ADMcG, Rice NSC. The success of penetrating keratoplasty for keratoconus. Eye 1990;4:673-88.

12. Girard LJ, Eguez I, Eśnaola N, Barnett L, Maghraby A. Effect of penetrating keratoplasty using grafts of various sizes on keratoconic myopia and astigmatism. J Cataract Refract Surg 1988;14:541-7.

13. Girard LJ, Esnaola N, Rao R, Barnett L, Rand WJ. Use of grafts smaller than the opening for keratoconic myopia and astigmatism: a prospective study. J Cataract Refract Surg 1992; 18:380-4.

14. Cohen KL, Holman RE, Tripoli NK, Kupper LL. Effect of trephine tilt on corneal button dimensions. Am J Ophthalmol 1986;101:722-5.

15. Tilanus M, van Rij G. An experimental comparison of three methods for trephination of the cornea and the consequent variations in the configurations of the trephine openings. Graefes Arch Clin Exp Ophthalmol 1987;225:50-2.

16. van Rij G, Waring GO III. Configuration of corneal trephine opening using five different trephines in human donor eyes. Arch Ophthalmol 1988:106:1228-33.

17. Troutman RC, Swinger CA, Belmont S. Selective positioning of the donor cornea in penetrating keratoplasty for keratoconus: postoperative astigmatism. Cornea 1984;3:135-9.

18. Krumeich J, Binder PS, Knulle A. The theoretical effect of trephine tilt on postkeratoplasty astigmatism. CLAO J 1988;14:213-9. 